\title{
Interpolation Coding: A Representation for Numbers in Neural Models
}

\author{
Dana H. Ballard \\ Department of Computer Science \\ The University of Rochester \\ Rochester, NY 14627 \\ TR 175 \\ May 1986 \\ (Preliminary Version)
}

\begin{abstract}
A central task of perception can be defined as one of computing hierarchies of invariants. One way of representing such invariants in intermediate levels of abstraction in this hierarchy is to use discrete units. These have been termed value units. A problem with such an encoding is that there has not been a good way to represent accurate numerical quantities using these units. This paper remedies the deficiency by describing a scheme that interpolates values between units representing fixed numerical quantities. The scheme has nice properties: it extends across functional mappings and it allows different sources of evidence to be combined.
\end{abstract}

This work was supported in part by the National Science Foundation under Grant DCR-8405720 and the National Institutes of Health under Public Health Service Grant 1R01NS22407-01. 


\section{Introduction}

Biologically plausible models of the brain's representation of perception and action require the specification of a task, followed by a detailed model of how the brain's components could be used to solve such a task. Our principal hypothesis is that: a major function of the perceptual system is to compute collections of invariants at different levels of abstraction. The value of computing these invariants is that they are increasingly concise descriptions of the relationships of the environment with respect to the agent. At the most abstract levels, this means that the agent can plan actions in a concise space of possibilities. Also, as the invariants become more abstract, they are true for larger portions of space and time. Thus they give the agent time to consider more elaborate actions.

An entity may be described as an invariant with respect to its component parts. Thus, to take an example from vision, an optic flow vector is an invariant with respect to certain spatiotemporal changes in the image function. A particular value of egomotion is invariant with respect to a collection of optic flow fields. Our concern is with invariants that can be described with a small number of parameters, typically less than ten. For example, rigid motion can be described by six parameters: a rotation (three) about an instantaneous center (three). The usefulness of small-parameter descriptions that describe a large number of different situations is primarily computational. Small parameter descriptions have been shown to be easily computed by distributed network models. These models have been termed connectionist to denote that the fundamental description of the problem is in the particular connections in the network. Previous papers have suggested a model for the visual system in terms of representations and computational strategies that make the computation of invariants efficient [Feldman and Ballard, 1982; Ballard, 1986; 1984; Feldman, 1985].

\subsection{The Value Unit Principle}

We have picked a specific representation first suggested by Barlow [1972] that is geared to solving parallel processing problems. This representation is termed value units. Value units are a general way of representing different kinds of multi-dimensional variables and functions without requiring that each unit have a large bandwidth. Value units break up the ranges of a variable into intervals and represent each interval with a separate unit. These intervals can be organized in many different ways. One straightforward way is to represent a variable $\mathbf{v}=\left(v_{1}, \ldots, v_{k}\right)$ isotropically by allocating a unit for each of $N^{k}$ discrete values. These values are the center of intervals of width $\Delta \mathbf{v}\left(\Delta v_{1}, \ldots, \Delta v_{k}\right)$. The value $k$ is the dimensionality of the variable. We will use the term parameter to refer to a scalar component of a variable, i.e., one of the $v_{i}$.

Let us compare the value unit encoding with variable representation in conventional Von Neumann computers. In a Von Neumann machine, variables only access one value at any instant, and acquire these values by assignment statements. For example, $x:=3 ; y:=4$ assigns values 3 and 4 to $x$ and $y$, respectively. Since a sequential computer can only access one value of a variable 
at a time, the notion of unique values for each variable at any instant is particularly appropriate. However, a parallel computer typically requires access to many values of a variable at the same time, and thus requires a different encoding scheme. A value unit representation such as an array of possible $(x, y)$ values allows this parallel access. This difference is shown in Figure 1 ( $a$ and $b$ ).

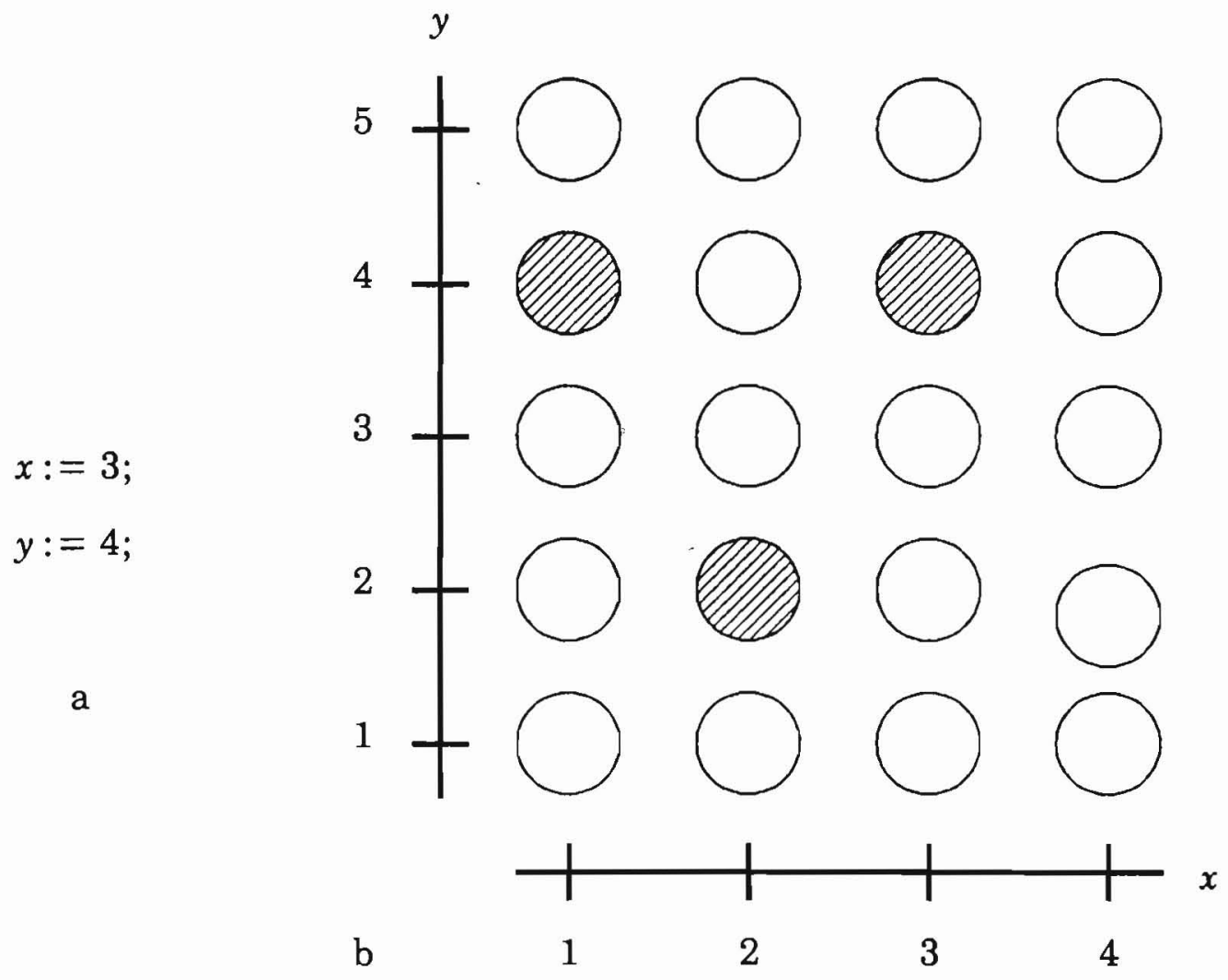

Figure 1: (a) Von Neumann encoding assigns a single value to a variable at any instant. (b) In contrast, value unit encoding allows many values of the same variable to be accessed simultaneously by having individual processors (units) for each of a discrete set of values. In the figure, in addition to $(3,4),(1,4)$ and $(2,2)$ are represented.

An important advantage of the value unit organization is that complex functions can be easily constructed, e.g., $f(x, y)=e^{V x} \cdot \Pi^{1 / y}$, are easily constructed by a table look-up strategy. Suppose one has such a function $f(x, y)$. Let us allot units for each interesting value of $x$ and have a similar set for the interesting $y$ values. One can think of these different values as very similar to just-noticeable-differences. Then the outputs of these units can be used pairwise to construct the function by connecting them to units representing appropriate values of $f$. We assume that both members of a pair of connections must be on before the unit representing a specific value of $f$ registers input. This type of input has been termed a conjunctive connection [Feldman and Ballard, 1982]. 


\subsection{Computing with Value Units}

A strong argument for value units is the facility with which they support parallel computation. To illustrate how value units can solve problems in parallel, we will describe the solution to a very specific problem. Consider the simple map in Figure 2a, with four regions. The problem is to color the map so that no two adjacent countries have the same color. Each region may be colored with one of the colors shown. This problem is representative of a ubiquitous class of problems which can be posed as: "satisfy the largest set of compatible constraints" [Freuder, 1978; Hummel and Zucker, 1983; Prager, 1980; Rosenfeld et al., 1976; Ullman, 1979]. When this problem is translated to value unit notation, the color of each region is a separate value unit. If a particular color is compatible with the currently chosen value units representing neighboring colors, then that unit is likely to be chosen to represent its corresponding region's color. The constraints are represented as links between units. There are many different ways to do this. We choose to let connections between locally incompatible colors be inhibitory (negative weights) and connections between compatible colors be excitatory (positive weights). These links are shown in Figure $2 \mathrm{~b}$. For brevity, two symmetric links are drawn as a single double-ended link. 

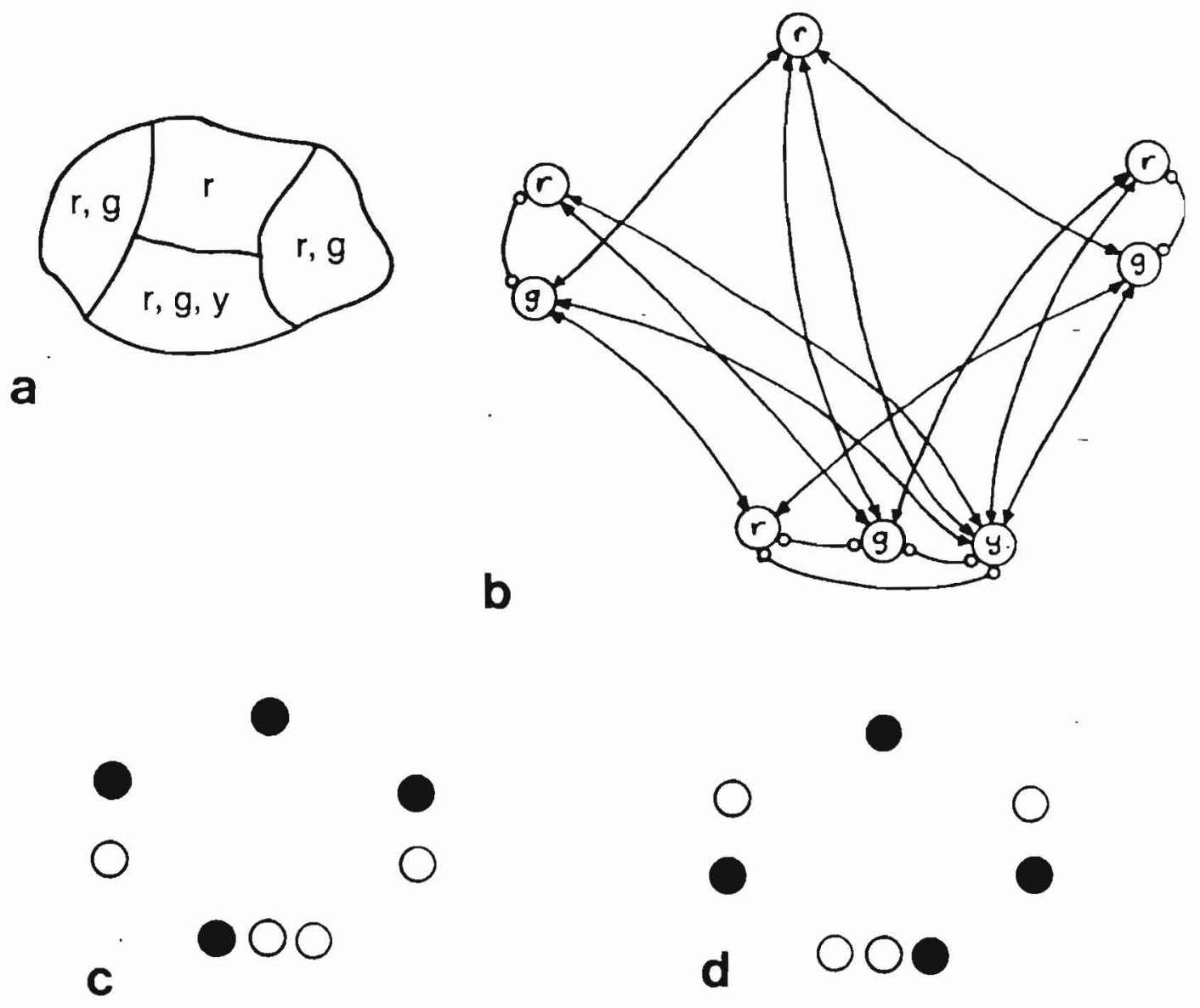

Figure 2: (a) A four-country map coloring problem: color the map with the colors shown ( $r=$ red, $\mathrm{g}=$ green, $\mathrm{y}=$ yellow) so that adjacent countries have different colors. (b) A value unit representation of the map coloring problem. A separate unit is allocated for each color of every country. Symmetric inhibitory connections are denoted by double-ended links terminating in small circles. Symmetric excitatory links are shown by double-ended links terminating in arrows. Other designs are possible; e.g., one could use inhibitory connections between incompatible colors in neighboring countries. The particular design keeps inhibition local to individual countries. (c) A particular starting configuration where dark units are on and light units are off. (d) The correct solution achieved by iteration using the state modification rule described in the text.

Networks of value units compute as follows. One can think of the $i$ th unit as having a small amount of information, $\left(s_{i}, \mathbf{w}_{i}\right)$, where $s_{i}$ is the state and $\mathbf{w}_{i}=$ $\left\{w_{i 1} \ldots w_{i n}\right\}$ is the synaptic weight vector. Where the weights are symmetric (i.e., $\left.w_{i j}=w_{i i}\right)$, there are several algorithms to minimize the "energy" functional

$$
E=-\sum_{i} \sum_{i} w_{i j} s_{i} s_{j}
$$


where $s_{i}$ is the binary state of a unit, either on $(0)$ or off $(1)$, and $w_{i j}$ is a real number that describes a particular constraint [Hopfield, 1982]. Adopting a technique developed by [Kirkpatrick et al., 1983], termed simulated annealing, Hinton and Sejnowski [1983] have provided an algorithm for finding the global minimum of Equation (1). Eventual convergence to a global minimum has been proved for a variant of this technique by [Geman and Geman, 1984]. In our example it is easy to spot check that the correct solution is an energy minimum by directly computing values of $E$ for different states, e.g.,

$$
\begin{aligned}
& E(r 1, g 2, b 3, g 4)=-10 \\
& E(r 1, g 2, b 3, g 3, g 4)=-8 \\
& E(\phi)=0 \\
& E(r 1, g 2, g 3, g 4)=-6
\end{aligned}
$$

This simple case can be handled with binary states and a gradient minimization rule:

where

$$
s^{k+1}:=1 \text { if } p_{i}^{k}>0 \text {, else } 0
$$

$$
p_{i}^{k}:=\sum_{j} w_{i j} s_{j}^{k}
$$

While we will not offer a formal proof that the above network converges, informally one can see that if the inhibitory links are weighted at least twice as great as excitatory links, the preponderance of excitatory links at the appropriate units will favor the solution described above. The example has been simulated and has proven stable to variations in the initial state.

The points behind the map coloring problem are threefold. First, the problem is characteristic of other constraint satisfaction problems in that empirical tests show that larger-scale versions do not require appreciably more time (although this has yet to be quantified). This controversial statement about problem scaling is currently based on empirical tests. Kirkpatrick et al. argue that convergence is based on how "frustrating" (incompatible) the constants are. The second point is that the kinds of constraints that we used are extremely general and can characterize a broad range of perceptual and cognitive situations [Hinton et al., 1984; Feldman, 1985; Ballard and Hayes, 1984]. In particular, problems in visual gestalt recognition can be described as trying to satisfy an appropriately weighted collection of local constraints [Ballard et al., 1983; Feldman, 1985]. The third lesson of constraint satisfaction is that local constraints can imply a global solution.

New learning algorithms have also recently been published that are also compatible with our formalism. These include supervised learning algorithms [Ackley et al., 1985; Rumelhart et al., 1985] and an associative learning algorithm [Rumelhart and Zipser, 1985]. 
The energy minimization can also be done with analog units [Hopfield, 1984; Hopfield and Tank, 1985]. The formulation with analog units allows the state to vary continuously in the interval $[0,1]$. Although the current algorithm with analog units is not guaranteed to find a global minimum, the limited testing that has been done shows that it produces good solutions for perceptual problems. The algorithm is:

\section{Algorithm Analog Energy Minimization}

State equations evolve according to

$$
\begin{aligned}
& d p_{i} / d t=-p_{i} / \tau+\Sigma w_{i j} s_{j} \\
& s_{i}=1 /\left(1+e^{\left.-p_{\imath}\right)}\right.
\end{aligned}
$$

where $\tau$ is a circuit time constant.

\subsection{Hierarchical Architectures}

The use of value units together with an energy minimization computational engine does not commit one to a hierarchical architecture, but for a host of reasons, some of which were mentioned earlier and others of which are developed in [Ballard, 1986; 1984], such organizations are desirable. The structure that we use and that is also advocated by others [Rumelhart and Zipser, 1985; Smolensky, 1986] contains well-defined layers of units, each having a given semantics, e.g., "optical flow units." Connections between layers have positive weights and connections within a layer have negative weights, as shown in Figure 3.

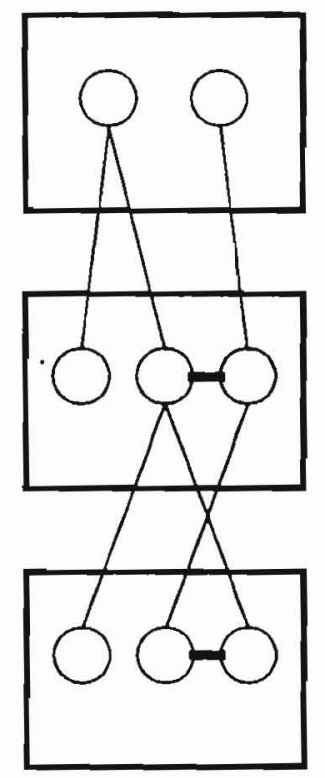

Figure 3: A hierarchical architecture. 


\section{Statement of the Problem and Solution}

We propose to crudely characterize the hierarchical structure in Figure 3 by associating it with the corresponding semantic hierarchy, as shown in Figure 4b. These tokens have qualitatively different mathematical properties, as in Figure 4c. At the most abstract level, the tokens represent discrete values of discrete processes, e.g., the color red for a particular country as captured by the map coloring example. The idealization of this representation is that its members can be characterized abstractly as a set of discrete elements. This model is also suited to the abstract entities in models of cognition, e.g., beliefs, plans, actions, relations, and objects. At the lowest level, the information is in the form of continuous values of continuous processes. The constraints at this level are captured by the classical Shannon sampling theorem and other more biologically-motivated variants. The idealization of this representation is that of continuous function of a set of variables, e.g., an image function $f(x, y, t)$.

In between these two extremes, we argue for an intermediate level that can be characterized as requiring continuous values of discrete processes. For example, the rotation of the visual field can be characterized by rotational values of a single rigid body motion process. There is only one process, but the actual parameter values that describe that process are numerical and vary over continuous intervals. Since this level is qualitatively different from the more abstract (discrete-discrete) and less abstract (continuous-continuous) levels, one might suspect that the mathematics required to model it would be qualitatively different also.

(a)

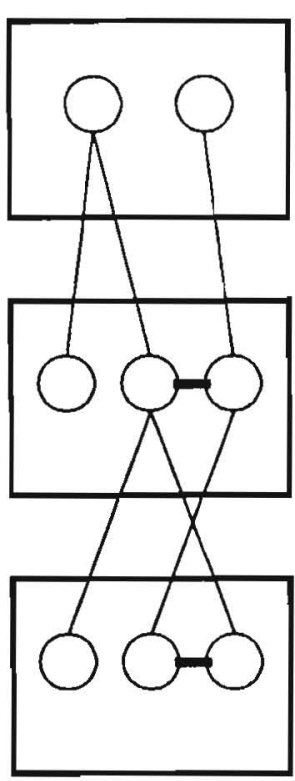

\begin{tabular}{|l} 
Beliefs \\
Plans \\
Actions \\
Relations \\
Objects \\
\\
Features \\
Spatially-Indexed Features \\
Retinotopically-Indexed \\
Features \\
\\
\\
Retinotopically-Indexed \\
Signal
\end{tabular}

Discrete Process Discrete Values

Discrete Process Continuous Values

(c)

Continuous Process Continuous Values

Figure 4: (a) A hierarchical architecture; (b) an interpretation of its possible semantics; and (c) mathematical characteristics. 
Although the values of a process in the intermediate level are continuous, the representation in which it is embedded is composed of discrete units. Here there seems at first to be a problem when accurate quantities are needed but only discrete values are represented. A standard solution to this problem has been to use a small grain size for the discrete range. The idea is that the samples will approximate the continuous values. However, as will be shown in a moment, this solution has technical problems in the presence of noise. A better solution, developed herein, is to interpolate between discrete values.

\subsection{A Delta Function Model}

To understand the need for interpolation, we first need to broadly characterize how the stimuli "look" at this level of representation. Our abstract model of the way data is acquired for an invariant represented as a parameter value in a parameter space is as follows. The general character of the world is that within a parameter space, for any perceptually relevant space-time interval, only a small distinct set of values will occur. These values may be idealized as points in the parameter space. The idealization is a model of the world. What is actually sensed is not the ideal point but a value that is usually corrupted by noise. This noise may arise from the world process itself or from sensor errors or both. This situation is described in Figure 5. In addition to these noise sources, there may be background noise from other world processes that only partially fit the parameter model. The main problem is to estimate a small set of values for the idealized model, given the noisy measurements.

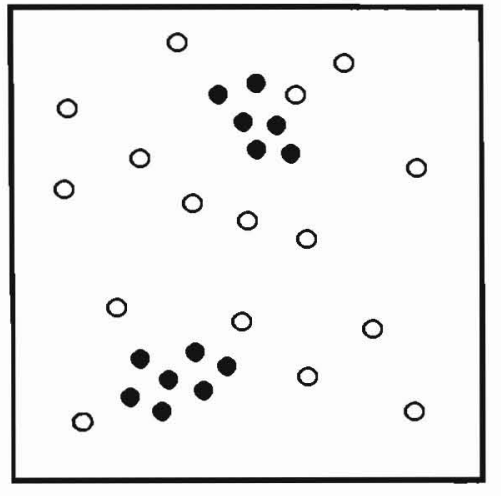

a. World

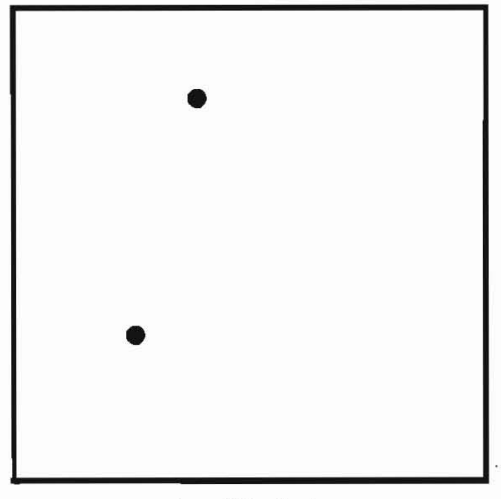

b. Model

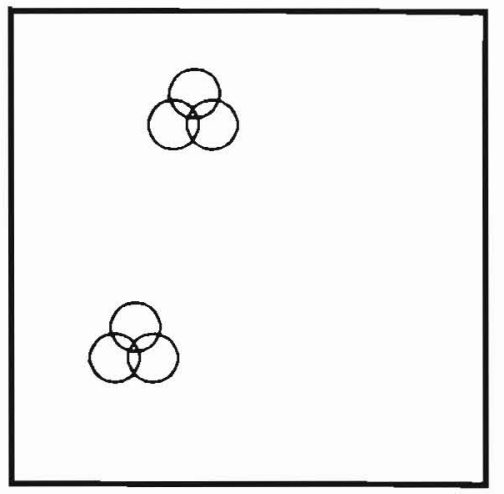

c. Internal Representation

Figure 5: Our description of the modeling problem for features (discrete processes, continuous values). An environment, as represented by sensors, contains background noise (open circles) and discrete processes (closed circles). The closed circles are scattered about a mean because of noise. The goal is to represent the central value of such processes, and remove background noise. Processes are interconnected in terms of networks, and because of an overall relaxation process, the central values may migrate.

This problem is made difficult both by the fact that the measurements are noisy and by the fact that the internal representation is discrete. The latter 
difficulty forces the use of units that are sensitive to the stimulus in an area that is generally referred to as its receptive field. Thus the internal representation approximates the ideal situation of the world model by interpolating across overlapping receptive fields, as shown in Figure 5c.

Since the model consists of sets of point processes, it is qualitatively different from the Shannon model and other models that regard the model as a continuous function over the domain. Instead, the model can be defined as a collection of delta functions, i.e.,

$$
\delta\left(x-x_{i}\right) \quad \text { for } i=1, \ldots M
$$

where $\delta(x)=\{1$ for $x=0$, and 0 for $x \neq 0\}$.

The internal representation represents this information in terms of projections into (Lagrangian) basis functions. Note that the signal is not bandlimited, yet we will be able to show that, under certain conditions, it can be unambiguously recovered.

\subsection{Interpolation and Minimization}

This qualitative description can be related to the formal models introduced earlier. Consider the equations for the analog model. In this system the potential $p_{i}$ and state $s_{i}$ change according to Equation (1.2). Let us simplify this equation and just look at the steady state value as $t \rightarrow \infty$. In this case

$$
p_{i}=\tau \sum_{j} w_{i j} s_{j}
$$

Notationally, to relate this model to value units, it is helpful to use the discrete value itself as the unit index instead of $i$. Thus for a discrete value of a parameter $x$ we will write $p\left[x_{i}\right]$ instead of $p_{i}$. Given this notation, we can make the following interpretation (that will be substantiated in latter development):

$$
\begin{aligned}
& s_{j}=s\left[x_{j}\right] \equiv \text { the "evidence for" measurement } x_{j} \\
& w_{i j}=w\left[x_{i}, x_{j}\right] \equiv \text { the weight required by the interpolation formula } \\
& \text { where } x_{j} \text { is evidence for } x_{i}
\end{aligned}
$$

\subsection{Interpolation and the Hough Transform}

Now let us return to the problem of estimating precise numerical values from discrete samples. The previous method for doing this used a truncation scheme [Duda and Hart, 1972; Ballard, 1984; Li et al., 1985] and can be described as follows. Given a set of measurements $x^{1}, \ldots, x^{k}$, quantize the space $X$ to some grain $\Delta x$. Then for each $x j, 1 \leq \mathrm{j} \leq \mathrm{k}$, round $x j$ to the nearest quantized value $x_{i}$ and add one to a counter associated with that value. If $p$ is an array representing the counters, the operations would be: 


$$
\begin{gathered}
x_{i}:=\operatorname{round}(x j) \\
p\left[x_{i}\right]:=p\left[x_{i}\right]+1 ;
\end{gathered}
$$

After this process, often referred to as voting (each measurement votes for its discrete value), the values with the most votes are selected as representative of the real world processes. The complete process is more complex, as the voters are in turn discrete processes who are themselves elected. Thus at some point this metaphor breaks down and the constraint satisfaction description from Section 1.2 must be invoked, but it still has intuition for local situations. This method can also be related to the steady state solution in Equation (2.1) by setting all the weights equal to one.

The primary problem with the rounding method is that it is tied to the grain size of the quantization. The value can only be reported to within plus or minus one grid unit. Furthermore, the following dilemma occurs. If the grain size is small to minimize the effects of roundoff error, then the individual measurements do not fall in the same quantized cell; whereas if the grain size is large, the roundoff error prohibits accurate localization. This problem is idealized in Figure 6. While these problems might be solvable at one level of abstraction, they can become much worse with the incorporation of hierarchies, since truncation errors can propagate up the hierarchies. Thus although there is a process centered at $(5,5)$ in $(a)$, it is not reported since the votes are all below a threshold. In (b), the process will be reported at $(6,6)$ or $(7,7)$ due to truncation error.

(a)

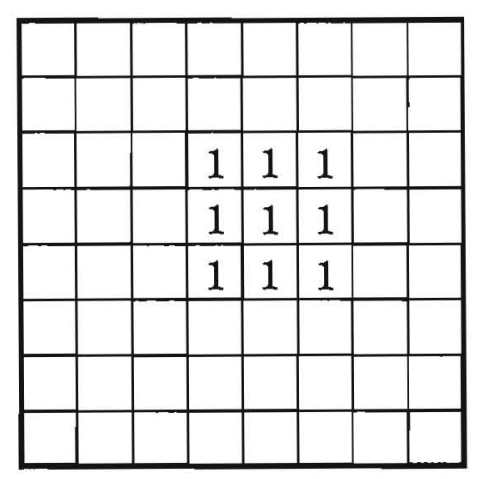

(b)

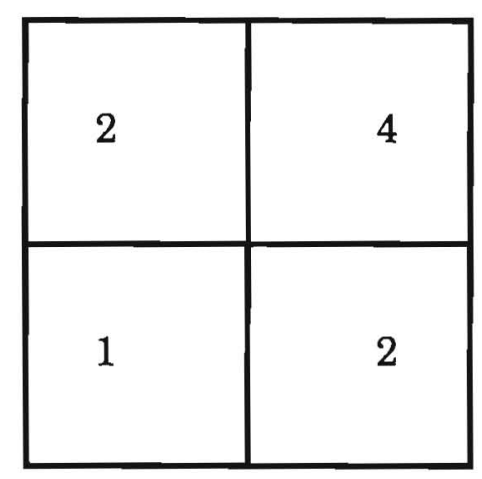

Figure 6: Characterization of the truncation problem: (a) at small grain sizes measurements are distributed; (b) at large grain sizes truncation errors are introduced. Numbers indicate votes, or the number of active, connected units.

One might try and fix this by using a very fine grid and convolving the measurements with a "peak sharpening" function [Brown, 1983]. This operation is expensive, however, in two. ways: (1) the fine-grained space is expensive to represent, requiring $N k$ elements, and (2) the convolution operation will cost $(M N)^{k}$ where $N$ is the linear size of the measurement space, $M$ is the 
diameter of the peak-sharpening function, and $k$ is the dimension of the stimulus.

Our solution is to not use rounding, but interpolation. The particular method is a form of Lagrangian interpolation [Davis, 1963; Jaeger and Starfield, 1974]. Instead of voting unity for a single discrete value, weighted votes are cast for each of $2^{k}$ neighboring values. The weights are chosen so that the weighted sum of the discrete values equals the original value. Figure 7 compares the two schemes for the two-dimensional case, i.e., $x=\left(x_{1}, x_{2}\right)$.

(a)

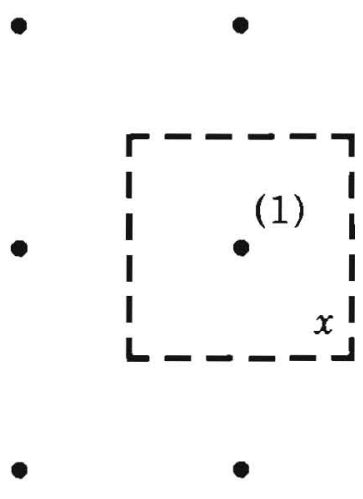

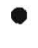
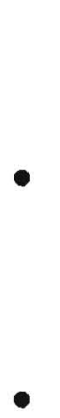

(b)

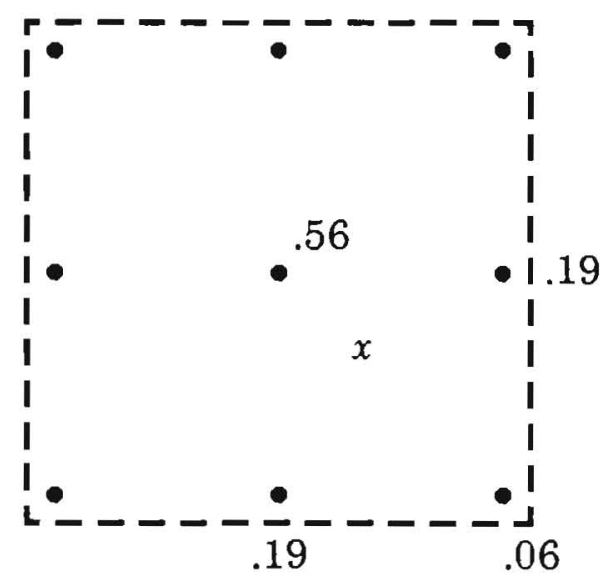

Figure 7: A comparison with the old truncation (a) vs. new interpolation (b) methods of value encoding. In the new method, receptive fields overlap and the value is distributed among neighboring units. Also, truncation errors are eliminated: in (a) the original value cannot be recovered, whereas in (b) it can.

This scheme has the following useful properties, which are developed formally in Section 3.

1) It is insensitive to random measurement noise. If the measurements are corrupted by noise of mean zero then the expected interpolated value is the same as the original value.

2) It can be generalized to parameter spaces of any dimensionality. However, we have argued that spaces of high dimensionality $(k>5-10)$ are infeasible mainly because they are too expensive to represent [Ballard, 1986].

3) If the mappings are locally linear over the grid size, i.e., have a Taylor series approximation that requires only first order terms, then the technique can be extended to handle the general case where both the voters and the votees are discretely represented.

4) The local linearity also allows the technique to be extended to general relations, i.e., multiple-valued functions. 
An important consequence of this model is that weights must be used to handle this encoding. For example, to continue the exposition in Figure 7b, a possible neuronal implementation of this model would connect the unit representing $x$ to the neighboring units that are shown in the figure with the appropriate weights. Since $x$ will also be represented with value units, these weights can be precomputed, as shown in Figure 8 .

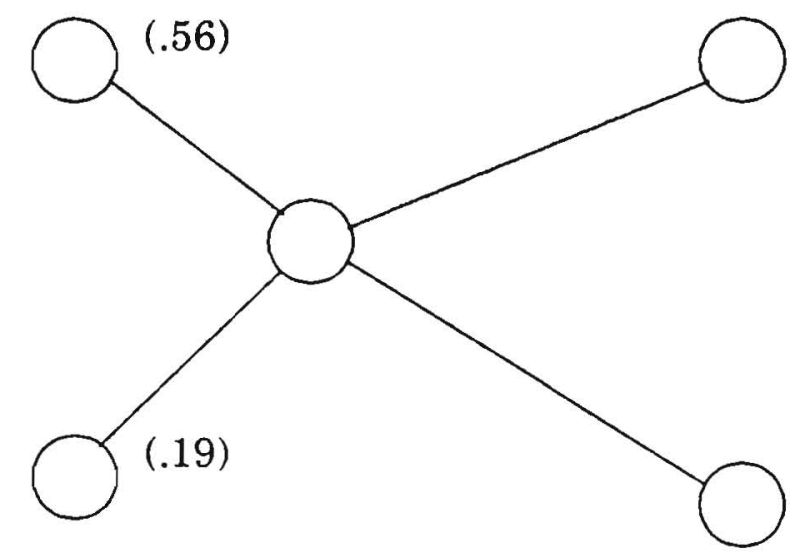

Figure 8: Weights for units can be precomputed based on the desired interpolation property.

One important consequence of this scheme is that the weights can no longer be symmetric. At present this means that there is no formal convergence theorem, but two ameliorating points are: (a) such a proof may be found for the special hierarchical architectures that we are considering; and (b) a limited number of simulations using nonsymmetric weights in such systems have demonstrated them to be stable.

\section{Properties of the Interpolation Scheme}

\subsection{Recovery and Noise Sensitivity}

The first property to show is that the interpolation scheme works, that is, given a scalar value $x$ and an interpolated estimate $x^{\prime}$, then in the noise-free case $x^{\prime}=x$. Suppose the value $x$ falls between discrete samples $x_{0}$ and $x_{1}$. Let $\Delta x=$ $x_{1}-x_{0}$. (In the multi-dimensional case to come, " $\Delta x$ " will be used in discussion for each dimension, loosely assuming isotropic sampling.) Also define

$$
\lambda\left(x ; x_{0}, x_{1}\right)=\left(x_{1}-x\right) /\left(x_{1}-x_{0}\right)
$$

and

$$
\mu\left(x ; x_{0}, x_{1}\right)=\left(x-x_{0}\right) /\left(x_{1}-x_{0}\right)
$$

Now associate a counter register $p\left[x_{i}\right]$ with the discrete value $x_{i}$. The registers are initialized to zero. Given $x$, the counters are updated by 


$$
\begin{aligned}
& p\left[x_{0}\right]:=p\left[x_{0}\right]+\lambda(x) \\
& p\left[x_{1}\right]:=p\left[x_{1}\right]+\mu(x)
\end{aligned}
$$

Note that $\lambda(x)$ and $\mu(x)$ can be precomputed for each of the discrete values of $x$. We will term the process specified by (3.3) and (3.4) as distributing the value of $x$. The original value can be estimated as

$$
x^{\prime}=\frac{p\left[x_{0}\right] x_{0}+p\left[x_{1}\right] x_{1}}{p\left[x_{0}\right]+p\left[x_{1}\right]}
$$

which in this case is

$$
x^{\prime}=\Lambda(x) x_{0}+\mu(x) x_{1}
$$

The relationship $x^{\prime}=x$ can be verified using direct substitution of (3.1) and (3.2). Let us denote the operation of distributing $x$ as $D(x)$ and that of interpolating as $I($ ). Then what has just been verified is that

$$
I(D(x))=x
$$

Similarly, one can show that these operations are linear in the general sense, i.e.,

$$
I\left(D\left(a_{1} x_{1}+a_{2} x_{2}\right)\right)=a_{1} x_{1}+a_{2} x_{2}
$$

This is an important property that we will use later.

To demonstrate noise insensitivity, consider a sequence of values $x^{1}, \ldots, x^{m}$ such that each value is an estimate of $x$ corrupted by noise of mean zero, i.e., $x j=$ $x+n j$, where $E(n)=0$. If we average the $x j$, this is a good estimate for $x$ since

$$
\frac{1}{m} \sum_{j=1}^{m} x^{j}=\frac{1}{m} \sum_{j=1}^{m} x+\frac{1}{m} \sum_{j=1}^{m} n^{j}=x+E_{m}(n)
$$

where $E_{m}(n)$, the average of $m$ noise values, $\rightarrow 0$ as $n \rightarrow \infty$. But this is also true for the interpolated estimate. For each measurement, the counters are updated using (3.3) and (3.4). At the end of this process the estimated value can be obtained from (3.5). Note that

$$
\Lambda^{j}=\left(x_{1}-x^{j}\right) /\left(x_{1}-x_{0}\right)
$$

so that

$$
p\left[x_{0}\right]=\sum_{j=1}^{m} \Lambda^{j}=\sum\left(x_{1}-x-n^{j}\right) /\left(x_{1}-x_{0}\right)
$$




$$
=\sum_{j=1}^{m}\left(x_{1}-x\right) /\left(x_{1}-x_{0}\right)-\sum n^{j} /\left(x_{1}-x_{0}\right)
$$

But in the limit the second term goes to zero, so that

$$
\sum_{j=1}^{m} \Lambda^{j}=m \Lambda
$$

A similar result holds for $P\left[x_{1}\right]$ so that, using (3.5),

$$
x^{\prime}=\frac{\lambda m x_{0}+\mu m x_{1}}{\lambda m+\mu m}=\Lambda x_{0}+\mu x_{1}
$$

What we have shown is that in the case of noise of zero mean the average of the interpolated estimate is the same as the average of the original data points.

While the model has the nice property of being insensitive to process noise, it has the undesirable property of being sensitive to background noise. (This was pointed out by Dave Sher.) This is easy to see, since background noise will have the effect of adding a constant to each of the units, thereby moving the estimate to a central point. This is not a disaster if one can estimate the background noise and use thresholds on each of the units to subtract this amount. However, it is still a step that will have to be taken.

\subsection{Generalization to K Dimensions}

Two dimensions will be handled first, to present the basic idea. Suppose now $x=\left(x_{1}, x_{2}\right)$. Let the discrete values surrounding the point $x$ have coordinates $\left(x_{10}, x_{20}\right),\left(x_{10}, x_{21}\right),\left(x_{11}, x_{20}\right)$, and $\left(x_{11}, x_{21}\right)$, as shown in Figure 9.

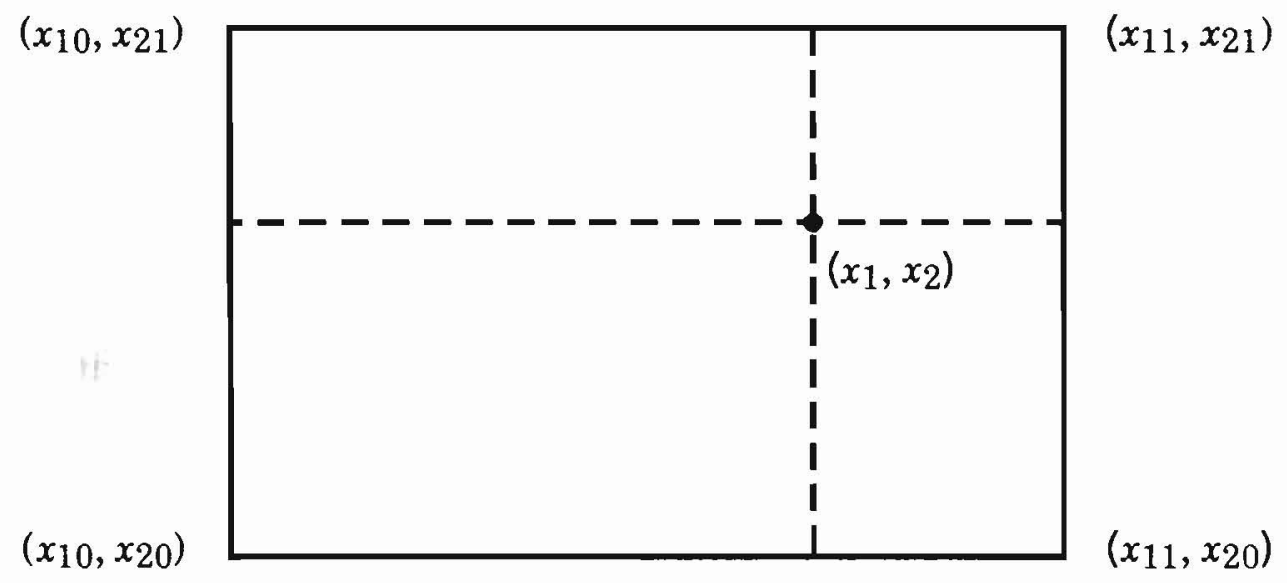

Figure 9: In two dimensions, the interpolation scheme is described in terms of the four neighbors of $x_{1} x_{2}$.

Now define 


$$
\begin{aligned}
& w_{10}=\left(x_{11}-x_{1}\right) /\left(x_{11}-x_{10}\right) \\
& w_{11}=\left(x_{1}-x_{10}\right) /\left(x_{11}-x_{10}\right) \\
& w_{20}=\left(x_{21}-x_{2}\right) /\left(x_{21}-x_{20}\right) \\
& w_{21}=\left(x_{2}-x_{20}\right) /\left(x_{21}-x_{20}\right)
\end{aligned}
$$

The algorithm for incrementing the counters becomes

Foreach $\left(x_{1}, x_{2}\right)$ do

1. Calculate $x_{10}, x_{11}, x_{20}, x_{21}$

2. Calculate $w_{10}, w_{11}, w_{20}, w_{21}$

3. Increment:

$$
\begin{aligned}
& p\left[x_{10}, x_{20}\right]:=p\left[x_{10}, x_{20}\right]+w_{10} \cdot w_{20} \\
& p\left[x_{10}, x_{21}\right]:=p\left[x_{10}, x_{21}\right]+w_{10} \cdot w_{21} \\
& p\left[x_{11}, x_{20}\right]:=p\left[x_{11}, x_{20}\right]+w_{11} \cdot w_{20} \\
& p\left[x_{11}, x_{21}\right]:=p\left[x_{11}, x_{21}\right]+w_{11} \cdot w_{21}
\end{aligned}
$$

Afterwards the estimated value $\left(x_{1}{ }^{\prime}, x_{2}{ }^{\prime}\right)$ can be calculated as

$$
\begin{aligned}
& p_{\mathrm{tot}}=p\left[x_{10}, x_{20}\right]+p\left[x_{10}, x_{21}\right]+p\left[x_{11}, x_{20}\right]+p\left[x_{11}, x_{21}\right] \\
&\left(x_{1}^{\prime}, x_{2}\right)=\left(p\left[x_{10}, x_{20}\right]\left(x_{10}, x_{20}\right)+p\left[x_{10}, x_{21}\right]\left(x_{10}, x_{21}\right)\right. \\
&\left.\quad+p\left[x_{11}, x_{20}\right]\left(x_{11}, x_{20}\right)+p\left[x_{11}, x_{21}\right]\left(x_{11}, x_{21}\right)\right) / p_{\mathrm{tot}}
\end{aligned}
$$

The above formulae are valid for the case when all the $x j$ fall within the same grid neighbors. This will not be the case in general, and the interpolation will have to be extended over more neighbors. For the case where the separation is large compared to the noise variance, i.e., $\Delta x>>\sigma_{x}$, one can use the eight neighbors together with the discrete value of $x$ for which $p[x]$ is a local maximum. Thus the two-dimensional case uses $3^{2}$ sample points for interpolation. Extending this scheme to $k$ dimensions requires $3 k$ neighboring values. In this case step 1 of the above algorithm must be replaced by:

$$
\text { for } \begin{aligned}
j & =1 \text { until } k \text { do } \\
x_{j 0} & =\left(x_{j} \operatorname{div} \Delta x_{j}\right) * \Delta x_{j} \\
x_{j 1} & =x_{j 0}+\Delta x_{j}
\end{aligned}
$$

Similarly, step 2 can be replaced by 


$$
\text { for } \begin{aligned}
j= & 1 \text { until } k d o \\
w_{j 1} & =\left(x_{j}-x_{j 0}\right) /\left(x_{j 1}-x_{j 0}\right) ; \\
w_{j 0} & =\left(x_{j 1}-x_{j}\right) /\left(x_{j 1}-x_{j 0}\right) ;
\end{aligned}
$$

Let $a_{j}$ be an index that takes on just 0 and 1 . Then step 3 becomes

$$
p\left[x_{1 \mathrm{a}_{1}}, \ldots, x_{k \mathrm{a}_{k}}\right]:=p\left[x_{1 \mathrm{a}_{1}}, \ldots, x_{k \mathrm{a}_{k}}\right]+\prod_{j=1}^{k} w_{j a_{j}}
$$

This completes our generalization of the algorithm to $k$ dimensions. Although the algebra is involved it can be shown that the noise resistance property that was demonstrated in Section 3.1 generalizes to the $\mathrm{k}$-dimensional case.

\subsection{Extension to Functional Mappings}

This section analyzes the issue of functional mappings. Suppose that $y=f(x)$. Under what conditions is it appropriate to distribute the values for $x$, map the distributed point set $\{x\}$ into $\{y\}$, and then interpolate $y$ from the distributed information? One would like the interpolated answer to be the same as that obtained by interpolating the distributed values for $y$. Formally this will be true if

$$
I(D(y))=I(D(f(D(x))))
$$

This is necessarily true if $f(x)$ can be regarded as a linear function for $x$ in the range $(x, x+\Delta x)$. Note that $f(x)$ does not have to be globally linear, but only locally linear. It can be shown formally in two steps. Let $y^{\prime}=f\left(x_{0}\right)$ and $y^{\prime \prime}=$ $f\left(x_{1}\right)$. The point $x$ is represented as $\lambda x_{0}+\mu x_{1}$. Furthermore, since $f(x)$ is linear, it can be described as $f(x)=a x+b$. Now one can verify that $\lambda y^{\prime}=\mu y^{\prime \prime}=y$ by direct substitution.

$$
\begin{aligned}
& \lambda\left(a x_{0}+b\right)+\mu\left(a x_{1}+b\right) \\
& \quad=a\left(\lambda x_{0}+\mu x_{1}\right)+b(\lambda+\mu) \\
& =a x+b=y
\end{aligned}
$$

The next question is, given $\lambda y^{\prime}+\mu y^{\prime \prime}=y$, does $I\left(\lambda D\left(y^{\prime}\right)+\mu D\left(y^{\prime \prime}\right)\right)=y$ ? But this follows directly from the linearity property (3.8), provided the grid sampling is such that $\Delta y / \Delta x \geq f\left(x^{\prime}\right)$.

Using the same arguments, it is easy to show that the mapping property generalizes to the case $y=f\left(x_{1}, \ldots, x_{k}\right)$. In certain instances the transformation $y=f\left(x_{1}, \ldots, x_{k}\right)$ is desired where portions of $\mathbf{x}$ are represented as non-overlapping subsets. That is, instead of a $k$-dimensional unit, the measurements are represented into $v$ subspaces of units where $x=\left(x_{1}, \ldots, x_{k l}, x_{k l+1}, \ldots, x_{2} k_{l}, \ldots\right.$,

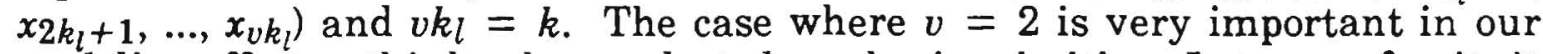
modeling effort as this has been selected as a basic primitive. In terms of units it has been described as a conjunctive connection [Feldman and Ballard, 1982]. In 
this case the weights for the $k$-dimensional space can be computed from those of the subspace by simply multiplying the appropriate combinations, as shown in Figure 10. The above generalization means that conjunctive connections can be used in this distributed interpolation scheme.

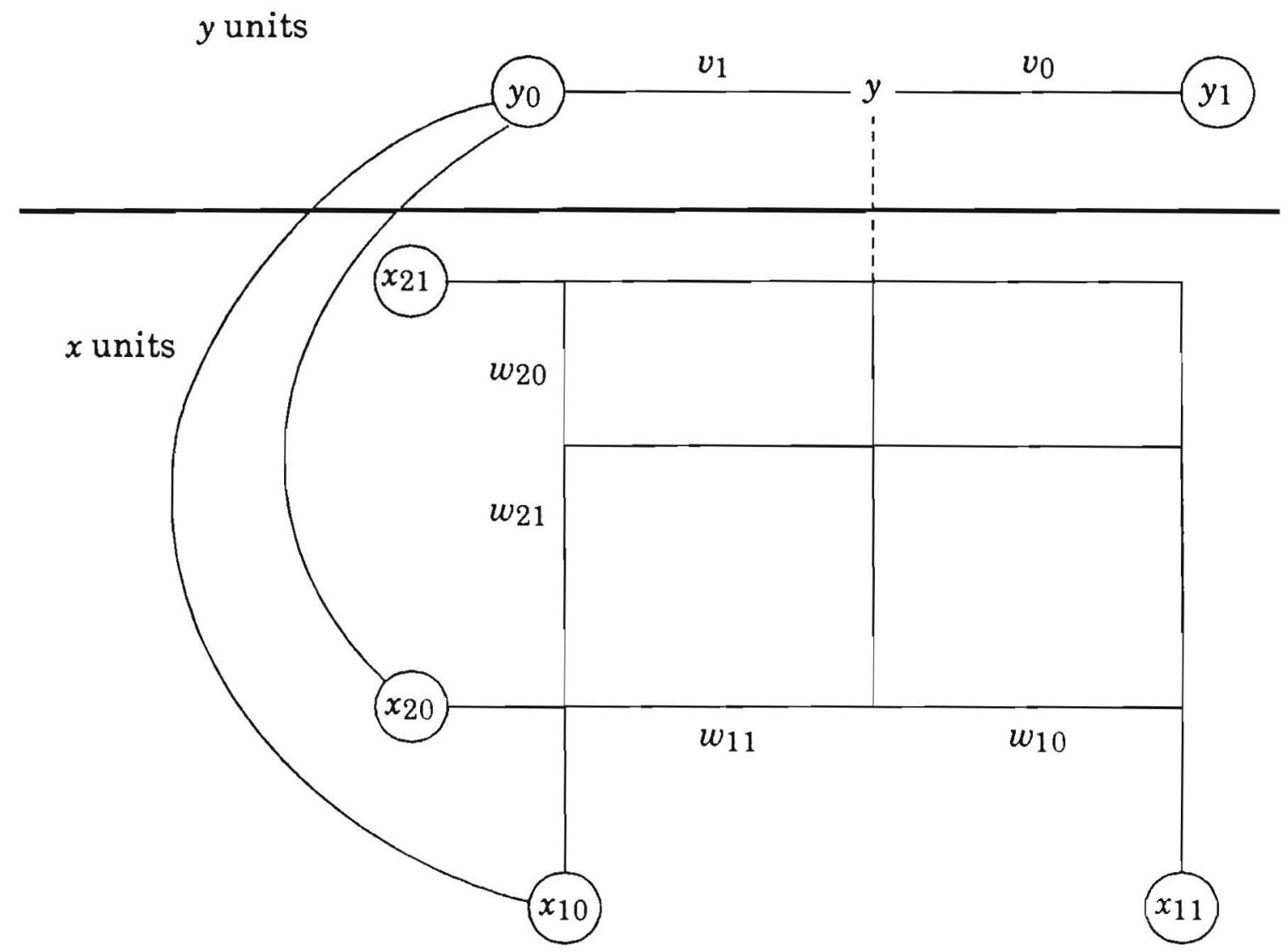

Figure 10: Combining measurements from subspaces. Only one of the four pairs of connections is shown.

\subsection{Extension to Relations}

Consider the problem where a constraint is specified by a linear functional, e.g., $a_{1} x_{1}+a_{2} x_{2}+a_{3} x_{3}+\ldots+a_{n} x_{n}=0$. In an application there might be $m$ of these equations in $n$ unknowns, where $m>>n$. Like the earlier cases, it is assumed that a subset of these equations are all derived from the same process, whereas others in the set are unrelated.

One way of handling this is via a general divide and conquer technique [Li et al., 1985]. For each cell, determine if the hyperplane passes through that cell. If the answer is yes, increment the counter associated with that cell. Cells with high counts are subdivided and the process is repeated. While this process is elegant, it suffers from the same deficiencies as the truncation technique mentioned in the introduction. 
The interpolation scheme can be extended to handle this case, but it is only practical for low-dimensional hyperplanes. To see how it works, consider the case of three-dimensional planes. Given $m$ planes of the form $a_{1}{ }^{i} x_{1}+a_{2}{ }^{i} x_{2}+$ $a_{3}{ }^{i} x_{3}+a_{4}{ }^{i}=0, i=1, \ldots, m$, the problem is to determine whether they have a common point of intersection. This can be handled hierarchically as follows. First intersect all pairs of planes to obtain lines; next intersect all pairs of lines to obtain points. The plane coefficients can be interpreted geometrically as a surface normal $\left(a_{1}, a_{2}, a_{3}\right)$ and a distance to the origin $a_{4}$ if the coefficients are first normalized so that $\sqrt{ }\left(a_{1}^{2}+a_{2}{ }^{2}+a_{3} 2\right)=1$. A line can be efficiently described by the equation $\mathbf{x}=\mathbf{D}+$ se where $\mathbf{e}$ is a unit vector in the direction of the line and $D$ is the vector of closest approach of the line to the origin. Given two planes, it is a straightforward algebraic task to calculate $(\mathbf{D}, \mathbf{e}) i j$ given two planes $\left(a_{1} i, a_{2} i, a_{3} i, a_{4} i\right)$ and $\left(a_{1} j, a_{2} j, a_{3} j, a_{4} j\right)$. Thus for each pair of planes, a three-dimensional line can be calculated and voted for. Given $N$ planes this results in $N^{2}$ line votes. These votes may or may not produce $N^{2}$ distinct lines, since nearby lines may interfere. Next, pairs of three-dimensional lines can be used to calculate three-dimensional points $\left(x_{1}, x_{2}, x_{3}\right)$, and these can be voted for.

While this method is sound in principle, its main practical drawback is that it is sensitive to noise. If the original planes all intersect at a point, then all pairs of lines will intersect at this point. However, if there are two sets of $N / 2$ planes, then there will be $N 4 / 16$ votes for each of the points of intersection and $7 N 4 / 8$ votes for "random" points. To take another example, if half the planes intersect at a point and the rest are "noise" planes, then there will be $N 4 / 16$ votes for the intersection point and $7 / 16 N^{4}$ votes for random points.

\subsection{Accuracy of the Representation}

We now tackle the question: how much numerical accuracy can be packed into the interpolation coding scheme? This can be modeled by assuming that each weight has $b$ bits and steady state, i.e., $s=0$ or 1 . In this case, the interpolation formula allows for $3 k b$ locations where $k$ is the dimension of the quantity represented. This is just a rough estimate, since not all of the locations are distinct. Figure 11 shows these locations for $k=2$ and $b=1$ and 2 .

What Figure 11 shows is that a huge amount of resolution is possible with very low accuracy in the weights. 


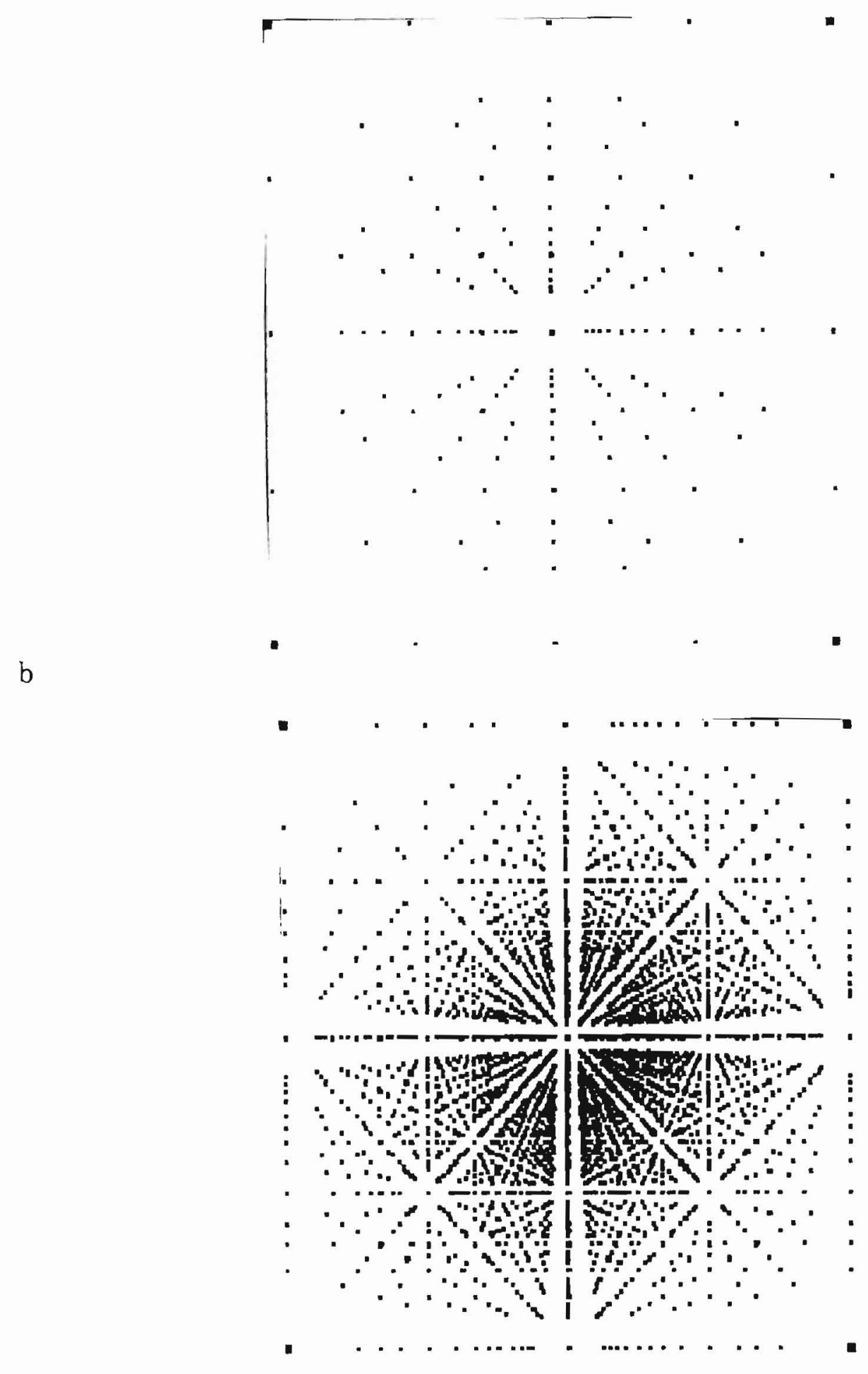

Figure 11: Interpolated points using different numbers of bits for the weights. (a) $b=1$ (each weight has value 1 or 0 ). (b) $b=2$ (each weight has one of four values: $1, .67, .33,0$ ). The interpolation uses values at the nine points shown with large filled squares. Only the values within a central square of side $\Delta x$ are important since at the periphery, the interpolation would use a different set of nine points. 


\subsection{Summary}

In this section we summarize the properties of the representation. Interpolation coding allows numerical data to be accurately represented as long as:

1) The constraints are locally linear with respect to the sampling grid. Thus an approximate upper bound on the grid spacing is determined by the validity of the linear terms in a Taylor series expansion in that neighborhood, i.e., $\Delta x<\Delta x_{\text {T.S. }}$.

2) The background noise is white. White noise effects all the grid points equally and can be removed by modeling its effects and subtracting them from the signal. This can be done by associating an appropriate threshold at each unit. Structure (non-white) noise will inevitably be interpreted as part of the signal.

3) The variance of the process noise is small compared to the sampling grid. This requirement follows from our recovery model that defines the measurements in terms of local units. This is not a strict criterion in the sense of (2), since the recovery model could be extended to use more units. It is in the form of lower bound, i.e., ideally $\sigma_{x}<<\Delta x$.

4) The inter-process spacing (IPS) should be greater than the sampling interval. Otherwise the measurements from two processes can be confounded. Thus $\Delta x<<\Delta x_{\text {IPS }}$.

5) Measurements are not effected by multiplicative scaling. This has been termed multiplicative invariance. Multiplying the update increment in (3.18) by an arbitrary factor does not change the value represented.

6) In the hierarchy, the measurement spacing should increase with the level in the hiaerarchy to take advantage of the fact that the number of processes at higher levels is usually less. Thus ideally, for levels 1 to $L$, $\Delta x_{1}<\Delta x_{2} \ldots<\Delta x_{L}$.

7) Ideally the accuracy is independent of the grid spacing. If the conditions (1) - (4) are satisfied, then the accuracy is only dependent on the number of bits used to represent the weights. This is a major improvement over truncation schemes.

\section{General Properties}

The formal properties of the interpolation coding model have already been discussed, but this discussion has taken place largely within the concept of a single array of units. To make these ideas relevant, they must be related to the computational engine discussed in Section 1.2. Also, there are two other less formally developed properties that have yet to be discussed: (1) evidence combination and (2) learning. Another issue that has been lightly discussed is 
the problems that arise when two processes are sufficiently close in values so as to interfere with each other. We begin with this issue.

\subsection{Sampling Theorem}

Section 2 introduced the notion of three qualitatively different levels of representation in the abstraction hierarchy. At the lowest level a function is represented by discrete samples and the classical Shannon sampling theorem describes the circumstances under which the samples can represent the function uniquely. If $F(\Omega)$, the Fourier transform of the function $f(x)$, is bandlimited, i.e., $f(\Omega)=0$ for $\Omega>\Omega_{\max }$ and the sampling interval $\Delta x$ such that $(1 / \Delta x) \leq 2 \Omega_{\max }$, then the samples uniquely represent the function. (There are some important details for the multidimensional case: see [Rosenfeld and Kak, 1976].)

In the case of a delta function model, the signal is definitely not bandlimited, so one must resort to a different kind of sampling theorem, but one that is very intuitive (and rather obvious). As long as different delta functions do not result in input to overlapping sets of units, then the samples uniquely represent the signal. Formally,

Sampling Theorem. Let the signal be $\delta\left(x-x^{i}\right), i=1, \ldots, M$. Represent this signal in terms of discrete basis functions according to (3.14) - (3.18). Let $d$ be the normalized minimum separation distance between any two basis functions, i.e., where $\Delta x_{l}$ is the separation distance of the $l$ th coordinate,

$$
d=\min _{i \neq j}\left\|\left(x_{l}^{i}-x_{l}^{j}\right) / \Delta x_{l}\right\|, \quad i, j=1, \ldots, M, \quad l=1, \ldots, k
$$

where $k$ is the dimension of the parameter vector. Then the signal can be unambiguously recovered if and only if

$$
d>2 \sqrt{ } k
$$

Proof (informal): The use of normalized distance transforms the parameter space to an isotropic discrete sampling of $R^{k}$ where the spacing between samples is unity. In this space, each impulse function is represented by samples in a hypercube of volume $2^{k}$. As long as no hypercubes intersect, then the signal can be recovered. The limiting case occurs when two hypercubes touch along a principal diagonal. Thus to prevent this from happening $d$ has to be greater than this distance.

\subsection{Computing with the Distributed Representation}

The properties of the representational scheme were discussed in Section 3.5. The key point of that discussion is that the representation can be analyzed in isolation from the computational process within which it is embedded. This means that the properties of the scheme as a data structure are independent, to a first approximation, from the computational process that uses the representation. Thus the weights of the updating function (3.18) can still be 
precomputed from the relations that the network represents, even when the state updating equations are complex.

The above point may not be intuitive, so we will elaborate it in some detail. For instance, what happens if this representation is used in the context of the algorithms described in Section 1.2? Importantly, by inspecting (3.18) it is evident that the properties we are concerned with are algorithm independent. This follows from the multiplicative invariance of the representation. In the ideal case, multiplying the increment in (3.18) by an arbitrary factor does not change the interpretation of the value represented. In keeping with the notation of Section 1.2, let us denote this factor as $s(t)$. Then equation (3.18) becomes:

$$
p\left[x_{\mathrm{la}_{1}}, \ldots, x_{k \mathrm{a}_{k}}\right]:=p\left[x_{\mathrm{la}_{1}}, \ldots, x_{k \mathrm{a}_{k}}\right]+\prod_{j=1}^{k} w_{j a_{j}} s
$$

From this one can see that the state of convergence can always be recovered by summing over the appropriate units. This formula is given by:

$$
s=\sum p\left[x_{1 a_{1}}, \ldots, x_{k a_{k}}\right]
$$

Thus the problems of defining an algorithm that converges, i.e., $s(t) \rightarrow s^{*}(t)$, are divorced from those that study the adequacy of the data structure, i.e., the properties derived in Section 3.

This has at least two implications as a biological model. In the first place, this can explain the difficulty of interpreting single-unit recordings. If the firing rate of a unit increases, in our model that can mean that either or both of the following has happened: (a) the confidence in the measurement has gone up, and/or (b) the value has shifted towards the value of that unit. The interpolation coding model implies that the situation can only be resolved by considering neighboring units.

\subsection{Evidence Combination}

One of the nicest features of the model is that it allows evidence from different sources to be combined. Consider the problem of estimating the depth of a retinotopic point $d(x, y)$. This can be captured by retinotopically indexed depth value units that represent depth either absolutely or relative to the fixation point (biological evidence favors the latter). In any case, many different processes are available to compute depth estimates for this point. Depth may be computed from: (i) stereo, (ii) motion, (iii) color, (iv) texture, and (v) shading, as well as other cues. Having a central representation solves the problem of how these measurements get combined. Although different methods of combination are possible, the most natural is to use the state, $s$, to express the confidence in that particular source. In that way the estimates will be combined in terms of a weighted average, owing to the linearity property. 
More specifically, consider two sources of information about the same parameter, $x$. Suppose that there are $n$ measurements from source 1 with associated strengths $s_{1}{ }^{j}$ and $m$ measurements from source 2 with associated strengths $s_{2} i$. Then according to the combination rule, the represented value of $x$ will be given by:

$$
x=\left(\sum_{j=1}^{n} s_{i}^{j} x^{j}+\sum_{i=1}^{n} s_{2}^{i} x^{i}\right) /\left(\sum_{j=1}^{n} s_{i}^{j}+\sum_{i=1}^{n} s_{2}^{i}\right)
$$

\subsection{Learning Algorithms}

An important general property follows from the multiplicative invariance that has implications for Hebbian learning rules: to preserve the interpolation property, if the weights are to be increased, they have to be increased by a multiplicative factor. Addition could be used only if one used weight variables that are logarithms of the weights herein; but the point is that, within this model, only these two options are available.

\subsection{Comparison with Coarse Coding}

Earlier we argued for qualitatively different representations of information in the abstraction hierarchy. If the information is in the form of discrete values of discrete samples, a different technique, termed coarse coding [Hinton, 1981; $1984]$ may be used. Coarse coding is based on intersecting receptive fields that are discrete supersets of an underlying set of discrete samples. Figure 12 shows the general idea.

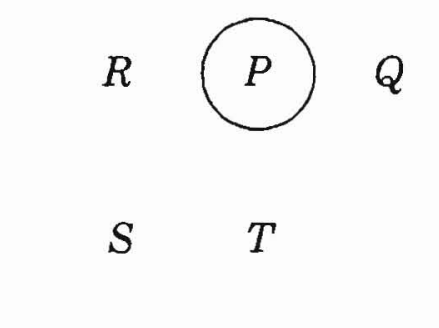

$$
\text { discrete encoding of } P \text { coarse coding of } P
$$

Figure 12: Comparing coarse coding and discrete coding. (a) Discrete coding uses one unit per entity. The presence of $P$ is signaled by a single unit. (b) Coarse coding uses more than one unit per entity. In this example the presence of $P$ is signaled by the collective firing of a $(S, P, T)$ unit and $\mathrm{a}(P, Q, R)$ unit. 
It is interesting to compare these two coding methods, even though they are models for different levels of abstraction. Table 1 shows the comparison of the two methods.

\begin{tabular}{|l|c|c|}
\hline & $\begin{array}{c}\text { Interpolation } \\
\text { Coding }\end{array}$ & $\begin{array}{c}\text { Coarse } \\
\text { Coding }\end{array}$ \\
\hline $\begin{array}{l}\text { Units Needed } \\
\text { to Interpolate }\end{array}$ & $D^{k}$ & $D$ \\
\hline $\begin{array}{l}\text { Savings in terms of } \\
\text { Fraction of Units }\end{array}$ & $1 / D^{k b}$ & $1 / D^{k-1}$ \\
\hline
\end{tabular}

Table 1: Comparison of Interpolation Coding and Coarse Coding. $D=$ overlap of receptive field; $k=$ dimension of stimulus; $b=$ number of bits in weight.

Both kinds of coding produce errors when the discrete process are sufficiently close so that the receptive fields overlap.

\subsection{Relationship to Biological Data}

The measurement of overlapping receptive fields in single unit recordings is a ubiquitous experience in experimental biology. Moreover, the parameters that these cells represent are most often modeled in numerical terms (e.g., [Andersen et al., 1985; Sakata et al., 1983].

Interpolation coding has a number of additional relationships with the details of these models. Recent anatomical measurements have strongly suggested that the neurons with visual, motor, and somatosensory cortex are hierarchically connected. For an extensive review of the visual cortex connections, see [Van Essen, 1986]. Also, the size of the receptive fields within these hierarchies increase with increasing level of abstraction in the hierarchy.

Another point of contact is in psychophysical measurements on humans that show that the measurement of a stimulus can be altered by the inclusion of a distractor stimulus of a different value. Perhaps the most well-known of these is the motion aftereffect illusion. In one set of experiments, subjects that adapt to motion in a pattern moving upwards at $30^{\circ}$ from north are then shown motion in the northerly direction. They erroneously report this as motion in the direction $-10^{\circ}$ from north [Levinson and Sekuler, 1976]. While the connection is somewhat fanciful at this point, this could be explained in terms of interpolation coding at the unit level. If for one reason or another a given measurement fatigues a cell that is part of the interpolation process, the represented measurement will move in the appropriate direction. 


\subsection{A More Biologically Plausible Linear Model}

The interpolation model of Section 3 uses orthogonal basis points. The advantage of this scheme is that it is easy to demonstrate most of the important properties of the representation related to interpolation and sampling. Furthermore, in a uniform sampling scheme, the weights can be easily computed by simple remainder operations. This model is not biologically plausible, however, in that it is unlikely that the precise placement of basis points can be guaranteed. Thus in this section we extend the model to the case where the points are randomly positioned. Besides being biologically plausible, this strategy has the advantage of needing much less points in the interpolation strategy.

Consider the situation shown in Figure 13 where the problem is to interpolate the point $\left(x_{1}{ }^{\prime}, x_{2}\right)$ from three non-colinear points $\left\{\left(x_{10}, x_{20}\right),\left(x_{11}\right.\right.$, $\left.\left.x_{21}\right),\left(x_{12}, x_{22}\right)\right\}$.

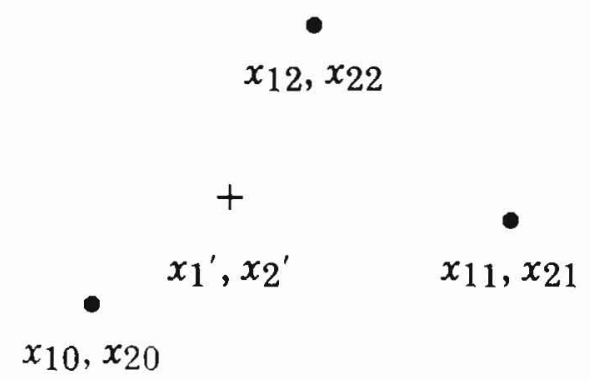

Figure 13: Three points are required to interpolate a two-dimensional value.

The problem is to determine a set of weights $w_{0}, w_{1}, w_{2}$ such that

$$
\begin{gathered}
w_{0} x_{10}+w_{1} x_{11}+w_{2} x_{12}=x_{1}{ }^{\prime} \\
w_{0} x_{20}+w_{1} x_{21}+w_{2} x_{22}=x_{2}{ }^{\prime} \\
w_{0}+w_{1}+w_{2}=1
\end{gathered}
$$

This can be easily solved. In general, for a $k$-dimensional space, $k+1$ points will be required. This is a huge savings over the $3^{k}$ points required by the orthogonal sampling strategy.

While at least $k+1$ points are necessary, it is unlikely that a biological system can be arranged to use exactly $k+1$ points. Hence the motivation for exploring the case where the number of sample points $P$ exceeds $k+1$. In this case the system is underdetermined, and some additional constraint must be found to allocate the extra degrees of freedom.

One way to do this would be to pick the weights so as to minimize the error induced if one of the basis points should be removed from the set. Since the effect is that of a moment, one can minimize the measure 


$$
E=\sum_{i=1}^{P} w_{i}^{2} d_{i}
$$

where $d_{i}=\left\|\mathbf{x}-\mathbf{x}_{i}\right\|$, subject to the constraint that $X \mathbf{w}=\mathbf{x}^{\prime}$ where $\mathbf{w}=$ $\left(w_{0}, w_{1}, w_{2}\right)$ and $\mathbf{x}^{\prime}=\left(x_{1}, x_{2}, 1\right)$.

As an example of how this works, consider the one-dimensional problem with three points $\left(x^{1}, x^{2}, x^{3}\right)$. Using the technique of Lagrange multipliers, the problem becomes:

$$
\min \sum_{i=1}^{3} w_{i}^{2} d_{i}+\Lambda_{1}\left(\sum_{i=1}^{3} w_{i}+1\right)+\Lambda_{2}\left(\sum_{i=1}^{3} w_{i} x^{i}+x\right)
$$

which has the following condition for a minimum:

$$
\left[\begin{array}{ccccc}
d_{1} & 0 & 0 & 1 & x^{1} \\
0 & d_{2} & 0 & 1 & x^{2} \\
0 & 0 & d_{3} & 1 & x^{3} \\
1 & 1 & 1 & 0 & 0 \\
x^{1} & x^{2} & x^{3} & 0 & 0
\end{array}\right]\left[\begin{array}{l}
w_{1} \\
w_{2} \\
w_{3} \\
\lambda_{1} \\
\lambda_{2}
\end{array}\right]=\left[\begin{array}{l}
0 \\
0 \\
0 \\
1 \\
x
\end{array}\right]
$$

This system has the solution:

$$
\begin{aligned}
& \lambda_{2}=X / D \\
& \Lambda_{1}=\left(A-B \Lambda_{2}\right) / A \\
& w_{i}=-\left(\Lambda_{1}+x^{i} \lambda_{2}\right) / d_{i} \quad \text { for } i=0,1,2
\end{aligned}
$$

where

$$
\begin{aligned}
& D=\sum_{i=1}^{3}\left(x^{i}\right)^{2} / d_{i} \\
& A=-\sum_{i=1}^{3} 1 / d_{i}
\end{aligned}
$$

and

$$
B=-\sum_{i=1}^{3} x^{i} / d_{i}
$$

This solution is meant to be illustrative of the general case where $x^{i}=$ $\left(x_{1 i}, x_{2 i}, \ldots, x_{k i}\right)$, in which there will be $(k+1)$ Lagrange multipliers needed to develop a set of $P+k+1$ equations in $P+k+1$ unknowns. 


\section{Summary}

The information required for perception and action forms a natural hierarchy. As one progresses up this hierarchy the mathematical characteristics change. At the lowest level, the information can be modeled as a continuous signal over space and time. At the highest level, the information is best thought of in terms of discrete entities. We have argued for an intermediate level of representation, consisting of continuous valued discrete processes. This fits naturally between the signal and symbol levels in the hierarchy.

This formulation leads naturally to the concept of interpolation coding, whereby the continuous values of the processes can be encoded into a discrete grid. This paper has developed the mathematical properties of such a representation, and related it to neurobiological and psychophysical data. These connections are tenuous at this point, and much additional work will be required to develop detailed comparisons. The important point is that the mathematical properties of this kind of encoding scheme make it extremely attractive.

\section{Acknowledgements}

This work benefited from many comments made by the Rochester Vision Research group, especially Horst Greilich, Tom Olson, and Dave Sher. Thanks also belong to Jerry Feldman, Paul Chou, and Lucy Lin for critiquing earlier drafts of the manuscript. Special thanks go to Peggy Meeker for preparing the figures and text. 


\section{References}

Ackley, D.H., G.E. Hinton, and T.J. Sejnowski, "A learning algorithm for Boltzmann machines," Cognitive Science 9, 1, 147-169, January-March 1985.

Andersen, R.A., G.K. Essick, and R.M. Siegel, "The encoding of spatial location by posterior parietal neurons," Science, 1985.

Ballard, D.H., "Cortical connections and parallel processing: structure and function," TR 133, Computer Science Dept., U. Rochester, revised January 1985; to appear, Behavioral and Brain Sciences, 1986.

Ballard, D.H., "Parameter nets," Artificial Intelligence 22, 235-267, 1984.

Ballard, D.H. and P.J. Hayes, "Parallel logical inference," Proc., Sixth Annual Conf. of the Cognitive Science Society, 286-292, Boulder, CO, June 1984.

Ballard, D.H., G.E. Hinton, and T.J. Sejnowski, "Parallel visual computation," Nature 306, 5938, 21-26, 3 November 1983.

Barlow, H.B., "Single units and sensation: a neuron doctrine for perceptual psychology?" Perception 1, 371-394, 1972.

Brown, C.M., "Inherent bias and noise in the Hough transform," IEEE Trans. Pattern Analysis and Machine Intelligence PAMI 5, 5, 493-505, September 1983.

Davis, P.J. Interpolation and Approximation. Blaisdell Publishing Co., 1963.

Duda, R.O. and P.E. Hart, "Use of the Hough transform to detect lines and curves in pictures," Commun. ACM 15, 11-15, January 1972.

Feldman, J.A., "Four frames suffice: a provisional model of vision and space," Behavioral and Brain Sciences 8, 265-289, June 1985.

Feldman, J.A. and D.H. Ballard, "Connectionist models and their properties," Cognitive Science 6, 205-254, 1982.

Freuder, E.C., "Synthesizing constraint expressions," CACM 21, 11, 958-965, November 1978.

Geman, S. and D. Geman, "Stochastic relaxation, Gibbs distributions, and the Bayesian restoration of images," IEEE Trans. PAMI 6, 6, 721-741, November 1984.

Hinton, G.E., "Distributed representations," Technical Report, Computer Science Dept., Carnegie-Mellon U., 1984.

Hinton, G.E., "Shape representation in parallel systems," Proc., 7th Int. Joint Conf. on Artificial Intelligence, 1088-1096, Vancouver, BC, Canada, 1981.

Hinton, G.E. and T.J. Sejnowski, "Optimal perceptual inference," Proc., IEEE Computer Vision and Pattern Recognition Conf., 448-453, Washington, DC, 1983. 
Hinton, G.E., T.J. Sejnowski, and D.H. Ackley, "Boltzmann machines: constraint satisfaction networks that learn," TR CMU-CS-84-119, Computer Science Dept., Carnegie-Mellon U., May 1984.

Hopfield, J.J., "Neural networks and physical systems with emergent collective computational abilities," Proc., National Academy of Sciences USA 79, 2554-2558, 1982.

Hopfield, J.J., "Neurons with graded response have collective computational properties like those of two-state neurons," Proc., Natl. Acad. Sci. 81, 3088-3092, May 1984.

Hopfield, J.J. and D.W. Tank, "'Neural' computation of decisions in optimization problems," to appear, Biological Cybernetics, 1985.

Hummel, R. and S. Zucker, "On the foundations of relaxation labeling processes," TR 80-7, McGill U., Montreal, 1980; IEEE Trans. Pattern Analysis and Machine Intelligence, 1983.

Jaeger, J.C. and A.M. Starfield. An Introduction to Applied Mathematics. Oxford Press, 1974.

Kirkpatrick, S., C.D. Gelatt, Jr., and M.P. Vecchi, "Optimization by simulated annealing," Science 220, 4598, 671-680, 1983.

Levinson, E. and R. Sekuler, "Adaptation alters perceived direction of motion," Vision Research 16, 779-781, 1976.

Li, H., M.A. Levin, and R.J. LeMaster, "Fast Hough transform," Proc., 3rd Vision Workshop, Bellaire, MI, October 1985.

Prager, J.M., "Extracting and labeling boundary segments in natural scenes," IEEE Trans. Pattern Analysis and Machine Intell. 2, 1, 16-27, 1980.

Rosenfeld, A., R.A. Hummel, and S.W. Zucker, "Scene labelling by relaxation operations," IEEE Trans. Systems Man Cybernet. 6, 1976.

Rosenfeld, A. and A. Kak. Digital Image Processing. Academic Press, 1976.

Rumelhart, D.E., G.E. Hinton, and R.J. Williams, "Learning internal representations by error propagation," ICS Report 8506, Institute for Cognitive Science, U. California, San Diego, September 1985.

Rumelhart, D.E. and D. Zipser, "Feature discovery by competitive learning," Cognitive Science 9, 1, 75-112, January-March 1985.

Sakata, H., H. Shibutani, and K. Kawano, "Functional properties of visual tracking neurons in posterior parietal association cortex of the monkey," J. Neurophysiology 49, 6, 1364-1380, June 1983.

Smolensky, P., "Foundations of harmony theory: cognitive dynamical systems and the subsymbolic theory of information processing," in D.E. Rumelhart and J.L. McClelland (Eds). Parallel Distributed Processing: Explorations in the 
Microstructure of Cognition. Vol. 1: Foundations. Cambridge, MA: Bradford Books/MIT Press, 1986.

Ullman, S. Interpretation of Visual Motion. Cambridge, MA: MTT Press, 1979.

Van Essen, D.C. Functional Organization of Primate Visual Cortex. In Peters and Jones (Eds). Cerebral Cortex (Vol. 3). Plenum Press, 1986. 